\title{
Competencias en carreras de ingeniería. Modelo para evaluar niveles de adquisición y requerimientos del mercado de trabajo
}

\author{
Competencies in Engineering Careers. Model to Evaluate Levels of Acquisition \\ and Requirements of the Job Market
}

\section{Competências em carreiras de engenharia. Modelo para avaliar níveis de aquisição e exigências do mercado de trabalho}

\author{
Marta Susana Comoglio \\ Universidad Nacional de Lomas de Zamora, Argentina \\ mcomoglio@ingenieria.unlz.edu.ar \\ https://orcid.org/0000-0002-6386-5883
}

\section{Resumen}

En Argentina, los procesos educativos en el campo de la enseñanza de las ingenierías están transitando por diversos procesos que promueven innovaciones curriculares orientadas hacia el diseño de planes de estudio por competencias y a la definición de nuevos estándares de acreditación.

El Instituto de Investigaciones en Tecnología y Educación (IIT\&E) de la Facultad de Ingeniería de la Universidad Nacional de Lomas de Zamora (UNLZ), en el marco de una investigación educativa, desarrolló un instrumento para medir, en relación con la adquisición de competencias, las distancias entre las expectativas, la formación recibida y lo demandado por el mercado de trabajo.

El estudio se desarrolló desde la perspectiva de los modelos de base subjetiva que evalúan la calidad de los servicios, y a partir de un cuestionario ad hoc que se administró a graduados. Con el enfoque especificado y con los datos obtenidos, segeneraron dos índices estadísticos.

El presente artículo tiene como finalidad presentar un modelo que, a través de los indicadores de adecuación de la competencia (IAC) y los indicadores de desarrollo de la 


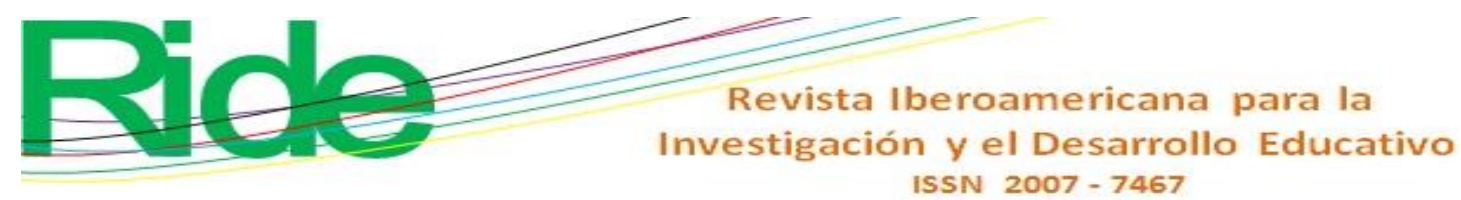

competencia (IDC), mide la magnitud y naturaleza del cambio que las instituciones enfrentan cuando inician procesos orientados a innovar curricularmente desde un plan de estudio por contenidos hacia otro por competencias.

Palabras clave: calidad, competencias, ingeniería, innovación curricular, modelo.

\section{Abstract}

In Argentina, educational processes in the field of engineering education are going through several processes that promote curricular innovations oriented towards the design of study plans by competences and the definition of new accreditation standards.

The Institute of Research in Technology and Education (IIT\&E) of the Faculty of Engineering of the National University of Lomas de Zamora (UNLZ), through an educational research, developed an instrument that allowed to measure, in relation to the acquisition of competences, the distances between the expectations, the training received, and the level of acquisition demanded by the job market.

The study was developed from the perspective of subjective models that evaluate the quality of the services, and froman ad hoc questionnaire that was administered to graduates, two statistical indexes were generated.

The purpose of this article is to present a model that, through the Adequacy of Competence (IAC) and Competence Development (IDC) indexes, measures the magnitude and characteristics of the change that institutions face when they initiate processes oriented to move from a curriculum for content to another by competencies.

Keywords: quality, competences, engineering, curriculum innovation, model. 


\section{Resumo}

$\mathrm{Na}$ Argentina, os processos educacionais no campo da educação em engenharia estão passando por vários processos que promovem inovações curriculares orientadas para a elaboração de planos de estudo por competências e a definição de novos padrões de acreditação.

O Instituto de Pesquisa em Tecnologia e Educação (IIT\&E) da Faculdade de Engenharia da Universidade Nacional de Lomas de Zamora (UNLZ), no âmbito de uma pesquisa educacional, desenvolveu um instrumento que permitiu medir, em relação à aquisição de competências, as distâncias entre as expectativas, a formação recebida e o nível de aquisição da demanda pelo mercado de trabalho.

O estudo foi desenvolvido a partir da perspectiva de modelos de base subjetivos que avaliam a qualidade dos serviços, e de um questionário ad hoc que foi administrado aos graduados dois índices estatísticos foram gerados.

O objetivo deste artigo é apresentar um modelo que, através dos Indicadores de Adequação da Concorrência (IAC) e Desenvolvimento da Competição (IDC), mede a magnitude e as características da mudança que as instituições devem fazer, quando começam processos orientados para inovar curricularmente, de um currículo de conteúdo para outro para competências.

Palavras- chave: qualidade, competências, engenharia, curriculum inovação, modelo.

Fecha Recepción: Julio 2018

Fecha Aceptación: Octubre 2018

\section{Introducción}

El propósito de este trabajo es presentar los resultados de un estudio cuyos datos fueron obtenidos a partir de la aplicación de un modelo diseñado para medir niveles de satisfacción respecto a la adquisición de competencias en carreras de ingeniería. A través de información proporcionada por dos indicadores que miden desde la perspectiva de los graduados y sus experiencias laborales la adecuación, superioridad o déficit entre las expectativas y los aprendizajes alcanzados, se aspira a contribuir con los procesos de innovación curricular. 


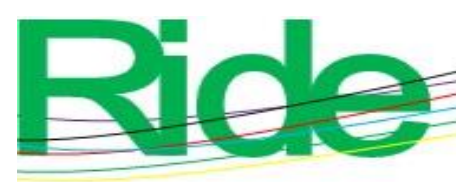

Revista Iberoamericana para la

Investigación y el Desarrollo Educativo

ISSN 2007 - 7467

La investigación se desarrolló en el ámbito de la Facultad de Ingeniería de la Universidad Nacional de Lomas de Zamora (UNLZ). En específico, los datos que se presentan son el resultado de una de las líneas de trabajo del Instituto de Investigaciones en Tecnología y Educación (IIT\&E). Cabe señalar que el IIT\&E es un centro asociado a la Comisión de Investigaciones Científicas de la Provincia de Buenos Aires (CIC-PBA), la cual, en el marco de sus funciones, lleva adelante las acciones de innovación curricular de la unidad académica.Yque el caso que se presenta es el resultado de su intervención en el proceso de modificación de estándares de acreditación de carreras de ingeniería que en Argentina se impulsan a partir del año 2015.

La declaración de Bolonia (Ministros Europeos, 1999) fue un punto de inflexión para los estudios universitarios, pues instó a garantizar la adquisición de competencias que aseguraran la inserción laboral de los estudiantes. Tal como lo señalan Herrero, González y Marín (2015) y Tejada (2012), por aquel entonces se comenzaron a explorar modelos que, además de conocimientos, promovieran el desarrollo de habilidades y actitudes. De esta manera, la perspectiva de formación por competencias adquirió centralidad en el nivel universitario.

De la Cruz Flores y Abreu Hernández (2014) afirman que la sociedad demanda sujetos que cuenten con pensamiento flexible, juicio crítico y reflexivo, y espera que su conocimiento sea utilizado para tomar decisiones, lo cual implica analizar, interpretar o realizar deducciones derivadas de una variedad de hechos y circunstancias. Por su parte, Ya-Hui, Li-Yia, Chao-Chin y Tzu-Ling (2012) subrayan que un aprendizaje acumulativo de conocimientos no resulta garantía de éxito profesional. Ambas afirmaciones llevan a reflexionar cómo la formación por competencias contribuye a la adquisición de aquellas capacidades y a mejorar la inserción profesional de los graduados.

En este contexto, en Argentina surge el Primer Acuerdo sobre Competencias (Consejo Federal de Decanos de Carreras de Ingeniería de la República Argentina [Confedi], 2006), el cual, siguiendo a Perrenoud (2013), sostiene que el saber hacer de los ingenieros es el resultado de una compleja estructura de conocimientos, habilidades y destrezas que requiere ser reconocida expresamente en el proceso de aprendizaje, y propone que las carreras de ingeniería se impartan a partir de diseños curriculares por competencias. Este enfoque implicó un desafío para el proyecto educativo de la Facultad de Ingeniería de la UNLZ; institución que en el año 2008 creó el IIT\&E 
con la misión de acompañar los procesos de innovación que tuvieran lugar en el marco de dicho acuerdo.

La propuesta del Confedi se estructuró a partir de la definición de unidades de competencias (Huerta, 2014) que se desagregaron en capacidades asociadas y componentes (Tobón, Pimienta y García, 2012 y Tobón, 2013), y dio como resultado una matriz que se presenta en la tabla 1.

Tabla 1.Competencias genéricas tecnológicas del perfil del ingeniero

\begin{tabular}{|c|c|}
\hline Competencias tecnológicas & Capacidades asociadas integradas \\
\hline $\begin{array}{l}\text { 1. Identificar, formular y resolver } \\
\text { problemas de ingeniería. }\end{array}$ & $\begin{array}{ll}\text { - } & \text { Identificar y formular problemas. } \\
\text { - } & \text { Realizar búsqueda creativa de soluciones y } \\
\text { seleccionar la alternativa más adecuada. } \\
\text { - } \\
\text { Implementar tecnológicamente una alternativa } \\
\text { de solución. } \\
\text { - Controlar y evaluar enfoques y estrategias } \\
\text { propios para abordar eficazmente la } \\
\text { resolución de los problemas. }\end{array}$ \\
\hline $\begin{array}{l}\text { 2. Concebir, diseñar y desarrollar } \\
\text { proyectos de ingeniería (sistemas, } \\
\text { componentes, productos o procesos). }\end{array}$ & $\begin{array}{l}\text { - } \quad \text { Concebir soluciones tecnológicas. } \\
\text { - } \quad \text { Diseñar y desarrollar proyectos de ingeniería. }\end{array}$ \\
\hline $\begin{array}{l}\text { 3. Gestionar, planificar, ejecutar y } \\
\text { controlar proyectos de ingeniería } \\
\text { (sistemas, componentes, productos o } \\
\text { procesos). }\end{array}$ & $\begin{array}{l}\text { - Planificar y ejecutar proyectos de ingeniería. } \\
\text { - } \quad \text { Operar y controlar proyectos de ingeniería. }\end{array}$ \\
\hline $\begin{array}{l}\text { 4. Usar de manera eficaz las técnicas } \\
\text { y herramientas de la ingeniería. }\end{array}$ & $\begin{array}{l}\text { - Identificar y seleccionar las técnicas y } \\
\text { herramientas disponibles. } \\
\text { - Usar y/o supervisar el uso de las técnicas y } \\
\text { herramientas. }\end{array}$ \\
\hline $\begin{array}{l}\text { 5. Contribuir a la generación de } \\
\text { desarrollos y/o innovaciones tecnológicas. }\end{array}$ & $\begin{array}{l}\text { - } \begin{array}{l}\text { Detectar oportunidades y } \\
\text { insatisfechas mediante }\end{array} \\
\text { tecnológicas. } \\
\text { - } \begin{array}{l}\text { Hacer un uso creativo de las tecnologías } \\
\text { disponibles. }\end{array} \\
\text { - } \\
\text { Emplear las formas de pensamiento } \\
\text { apropiadas para la innovación tecnológica. }\end{array}$ \\
\hline
\end{tabular}

Fuente: Confedi (2006) 


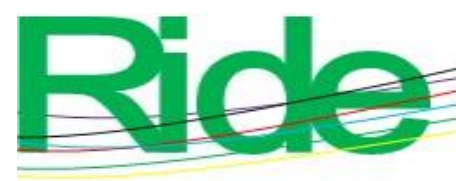

Revista Iberoamericana para la Investigación y el Desarrollo Educativo ISSN 2007 - 7467

A partir de la revisión global de estándares de acreditación que impulsa el Ministerio de Educación (Comisión Nacional de Evaluación y Acreditación Universitaria [Coneau], 2015), el acuerdo del Confedi del año 2006 recobró vigencia, ya que se aprueba un nuevo documento que propone que las futuras acreditaciones se desarrollen bajo planes de estudio diseñados por competencias (Confedi, 2017).

Pérez, Vilariño y Ronda (2017) afirman que toda innovación está condicionada por los alcances y profundidad de las directrices que la impulsan, pero también por los valores, creencias y prácticas cotidianas, las cuales definen la magnitud y orientación de las acciones; esta circunstancia llevó a que desde el IIT\&E se desarrollara un estudio diagnóstico sobre las carreras de la unidad académica y el contexto en el que se impartían.

Existe coincidencia entre los autores de que las políticas que institucionalizan procesos de acreditación, como así también los procesos de innovación curricular, se asocian a la mejora, por lo tanto, el IIT\&E optó por un enfoque de evaluación de la calidad para el estudio. Es así como, a partir de las recomendaciones de Duque Oliva y Chaparro Pinzón (2017) acerca de los métodos para evaluar la calidad de programas educativos, se consideró que aquellos que miden percepciones a través de juicios de satisfacción se ajustaban adecuadamente a los objetivos definidos por la institución.

Martínez, Blanco y Castán (2013) advierten que, en líneas generales, los procesos de calidad en los ámbitos educativos han tomado como punto de partida la hipótesis de que los responsables de las instituciones educativas están en condiciones de desarrollar programas orientados a la satisfacción del estudiante. Sin embargo, las actuales corrientes teóricas en este ámbito indican que lo relevante son las necesidades del usuario. Teniendo en cuenta que el principal usuario del servicio educativo es el alumno, se consideró la opinión del egresado como el insumo estratégico para los fines del presente trabajo. 


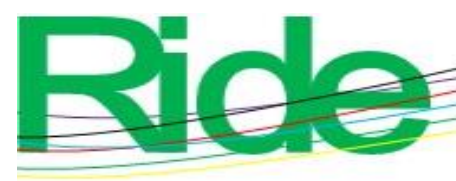

Revista Iberoamericana para la

Investigación y el Desarrollo Educativo

ISSN 2007 - 7467

\section{Método}

A partir de los diferentes métodos de base subjetiva desarrollados hasta la fecha, recogidos por Duque Oliva y Diosa Gómez (2014), se realizó una adaptación ad hoc que dio como resultado un estudio de diseño descriptivo y transeccional, sobre la base de una encuesta que centró la indagación en torno a las capacidades asociadas a la primera competencia genérica tecnológica definida por Confedi (2006). Por lo tanto, se consultó cómo los graduados percibían el nivel de desarrollo de la competencia "Identificar, formular y resolver problemas de ingeniería, como así mismo concebir, diseñar y desarrollar proyectos de ingeniería".A tales fines, se les preguntó cuál había sido, en relación con las capacidades y componentes asociados, el nivel de adquisición durante la carrera, cuál el aprendizaje esperado y cuáles las demandas de los empleadores. El instrumento recogió información cualitativa, pero, a efectos de que los datos pudieran recibir tratamiento estadístico, se requirió que las respuestas fueran volcadas en una escala Likert (1-10).

El cuestionario fue administrado durante el segundo semestre de 2017 deforma aleatoria a una muestra de 108 egresados de Facultad de Ingeniería de la UNLZ. Se consideró que los graduados con no más de cinco años de egreso resultaban ser actores clave. Debido a que su reciente condición de alumnos les permitía aportar información con buen criterio de confiabilidad acerca de su tránsito por la carrera, así como acerca de las experiencias relacionadas con su inserción laboral.

Los datos obtenidos recibieron tratamiento estadístico con el objeto de mesurar la distancia entre expectativas, formación recibida y demandas del mercado laboral. A partir de las medias obtenidas se construyeron dos índices: $a$ )Índice de Adecuación de las Competencias (IAC), que mide la relación percibida entre lo esperado y lo recibido por los graduados y $b$ )Índice de Desarrollo de las Competencias (IDC), que evalúa la percepción en relación con el nivel adquirido y lo requerido por el mercado laboral.

Para la determinación de esos índices, a las medias obtenidas en cada uno de los componentes de las capacidades asociadas se les aplicó el siguiente cálculo:

- $\quad \mathrm{IAC}=$ valor observado $(\mathrm{VO})$-valor esperado $(\mathrm{VE})$.

- $\quad$ IDC $=\mathrm{VO}$-valor requerido $(\mathrm{VR})$ 
A continuación, se presenta esquemáticamente el esquema conceptual a partir del cual se desarrolló el instrumento de medición (ver tabla 2).

Tabla 2. Competencia desagregada en capacidades asociadas y sus respectivos indicadores

\begin{tabular}{|c|c|}
\hline \multicolumn{2}{|c|}{$\begin{array}{c}\text { Competencia 1. Identificar, formular y resolver problemas de ingeniería, como así mismo } \\
\text { concebir, diseñar y desarrollar proyectos de ingeniería }\end{array}$} \\
\hline Capacidades asociadas & Capacidades componentes o indicadores \\
\hline Identificar y formular problemas. & $\begin{array}{ll}\text { - } & \text { Identificar una situación problemática. } \\
\text { - } & \text { Identificar y organizar los datos pertinentes a } \\
\text { un problema. } \\
\text { - } \quad \text { Evaluar el contexto particular del problema e } \\
\text { incluirlo en el análisis. } \\
\text { - Delimitar el problema y formularlo de manera } \\
\text { clara y precisa. }\end{array}$ \\
\hline $\begin{array}{l}\text { Realizar búsqueda creativa de } \\
\text { soluciones y seleccionar la alternativa } \\
\text { más adecuada. }\end{array}$ & 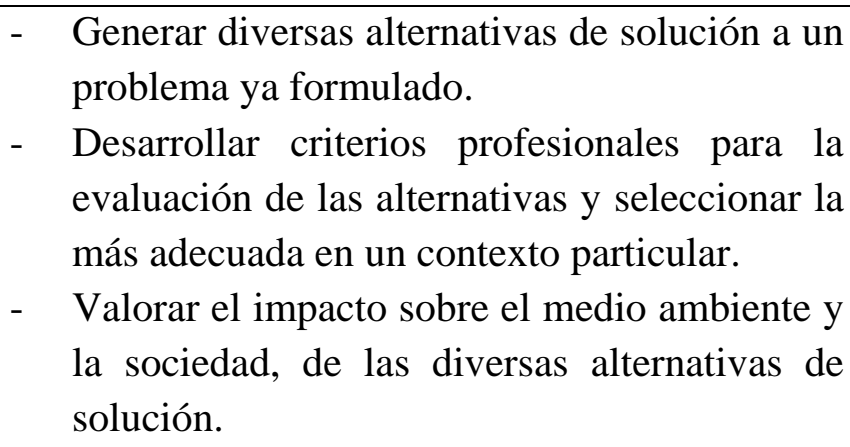 \\
\hline $\begin{array}{l}\text { Implementar tecnológicamente una } \\
\text { alternativa de solución. }\end{array}$ & $\begin{array}{l}\text { - } \begin{array}{l}\text { Realizar el diseño de la solucióntecnológica. } \\
\text { - }\end{array} \text {-ncorporar al diseño las dimensiones del } \\
\text { problema (tecnológica, temporal, económica, } \\
\text { financiera, medioambiental, social, etc.) que } \\
\text { sean relevantes en su contexto específico. } \\
\text { - Planificar la resolución (identificar el } \\
\text { momento oportuno para el abordaje, estimar } \\
\text { los tiempos requeridos, prever las ayudas } \\
\text { necesarias, etc.). } \\
\text { - Optimizar la selección y uso de los materiales } \\
\text { y/o dispositivos tecnológicos disponibles para } \\
\text { la implementación. } \\
\text { Elaborar informes, planos, especificaciones y } \\
\text { comunicar recomendaciones. } \\
\text { Controlar el proceso de ejecución. }\end{array}$ \\
\hline
\end{tabular}




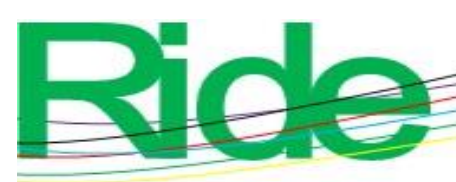

Revista Iberoamericana para la Investigación y el Desarrollo Educativo ISSN $2007-7467$

Controlar y evaluar enfoques y estrategias propios para abordar eficazmente la resolución de los problemas.
- Controlar el propio desempeño y saber cómo encontrar los recursos necesarios para superar dificultades.

- Establecer supuestos, de usar técnicas eficaces de resolución y de estimar errores.

- Monitorear, evaluar y ajustar el proceso de resolución del problema.

- Usar lo que ya se conoce; identificar lo que es relevante conocer, y disponer de estrategias para adquirir los conocimientos necesarios.

Fuente: Elaboración propia con base en Confedi (2006)

\section{Resultados}

En este apartado se presentan para cada uno de los componentes de la competencia analizada las medias correspondientes al VO, VE y VR. En segundo término, se presentan los índices IAC e IDC.

\section{Capacidad para identificar y formular problemas}

Al analizar la capacidad y sus componentes desagregados se observa que el aprendizaje con mayor nivel de adquisición demandado por el sector empleador fue "Delimitar el problema y formularlo de manera clara y precisa" (8.40), mientras que el que menor requerimiento obtuvo fue "Evaluar el contexto particular del problema e incluirlo en el análisis" (8.07). Desde la perspectiva de los graduados, este fue el componente con mayor expectativa de adquisición (7.87) (ver tabla 3). Por otra parte, el valor observado más alto fue "Identificar una situación problemática”, cuya media se ubicó en 8.56. 


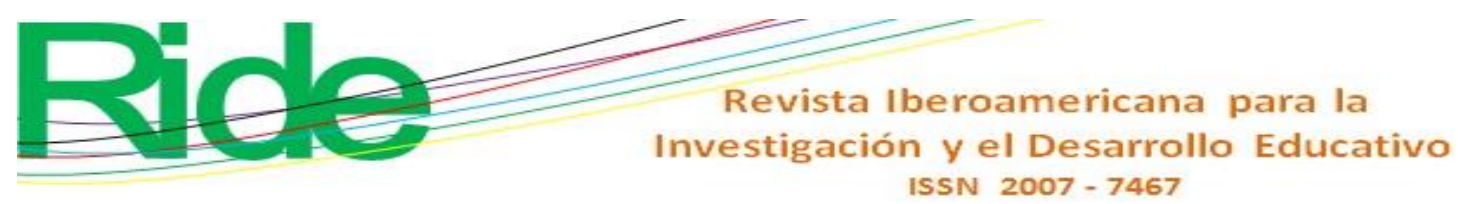

Tabla 3. VO, VE y VR para los componentes de la capacidad "Identificar yformular problemas"

\begin{tabular}{lccc}
\hline Componentes de la capacidad & $\boldsymbol{V O}$ & $\boldsymbol{V E}$ & $\boldsymbol{V R}$ \\
\hline $\begin{array}{l}\text { Identificar situación } \\
\text { problemática. }\end{array}$ & 8.56 & 7.44 & 8.15 \\
$\begin{array}{l}\text { Identificar y organizar los datos } \\
\text { pertinentes a un problema. }\end{array}$ & 7.75 & 7.47 & 8.33 \\
$\begin{array}{l}\text { Evaluar el contexto particular } \\
\text { del problema e incluirlo en el } \\
\text { análisis. }\end{array}$ & 7.55 & 7.60 & 8.07 \\
$\begin{array}{l}\text { Delimitar el problema y } \\
\text { formularlo de manera clara y } \\
\text { precisa. }\end{array}$ & 7.87 & 7.45 & 8.40 \\
\hline
\end{tabular}

Fuente: Elaboración propia

Capacidad de realizar búsqueda creativa de soluciones y seleccionar las alternativas más adecuadas

Al centrar el análisis en la capacidad para realizar búsquedas creativas de soluciones y seleccionar la alternativa más adecuada (ver tabla 4), el componente con mayor nivel de desarrollo requerido por el sector empleador fue "Generar diversas alternativas de solución a un problema ya formulado" (8.45), valoración que resultó ser consistente con lo percibido por los encuestados, cuyas respuestas ubicaron el valor observado por encima de los ocho puntos (8.29) y de esta forma resultó ser el componente con la ponderación más alta.

El componentecon mayor expectativa para esta capacidad fue "Valorar el impacto de las diversas alternativas de solución sobre el medio ambiente y la sociedad" (7.73), lo que resultó significativo en virtud de que fue el de más bajo nivel de requerimiento por el sector empleador (7.27). 


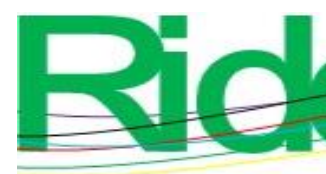

Revista Iberoamericana para la

Investigación y el Desarrollo Educativo

ISSN 2007 - 7467

Tabla 4. VO, VE y VR para los componentes de la capacidad "Realizar búsqueda creativa de soluciones y seleccionar la alternativa más adecuada"

\begin{tabular}{lccc}
\hline Componentes de la capacidad & VO & VE & VR \\
\hline $\begin{array}{l}\text { Generar diversas alternativas de solución a un problema ya } \\
\text { formulado. }\end{array}$ & 8.29 & 7.51 & 8.45 \\
$\begin{array}{l}\text { Desarrollar criterios profesionales para la evaluación de las } \\
\text { alternativas y seleccionar la más adecuada en un contexto } \\
\text { particular. }\end{array}$ & 8.00 & 7.04 & 8.16 \\
$\begin{array}{l}\text { Valorar el impacto sobre el medio ambiente y la sociedad, de } \\
\text { las diversas alternativas de solución. }\end{array}$ & 7.36 & 7.73 & 7.27 \\
\hline
\end{tabular}

Fuente: Elaboración propia

\section{Capacidad de implementar tecnológicamente una alternativa de solución}

Se observa que esta capacidad ha sido evaluada globalmente en forma positiva, ya que, para todos los componentes, las medias observadas resultaron superiores a sus correspondientes en materia de expectativas (ver tabla 5). Los niveles más altos observados resultaron ser para el componente "Elaborar informes, planos, recomendaciones y comunicarlos" (8.45), seguido de "Optimizar la selección y uso de materiales y dispositivos tecnológicos disponibles para la implementación" (8.44); de forma coincidente, el mercado de trabajo requirió mayor nivel de adquisición para los mismos componentes (8.71 y 8.53 respectivamente). Resulta interesante observar que el resultado del valor esperado para el componente "Realizar el diseño y modelado de una solución tecnológica" se ubicó en 5.93, lo cual es ampliamente superado tanto por el valor observado (7.51) como por nivel de adquisición requerido (7.69). 


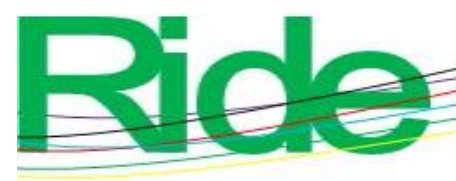

Revista Iberoamericana para la

Investigación y el Desarrollo Educativo

ISSN 2007 - 7467

Tabla 5. VO, VEy VR para los componentes de la capacidad "Implementar tecnológicamente una alternativa de solución"

\begin{tabular}{lccc}
\hline Componentes de la Capacidad & VO & VE & VR \\
\hline $\begin{array}{l}\text { Realizar el diseño y modelado de una solución tecnológica. } \\
\text { Incorporar a un diseño las dimensiones relevantes al contexto } \\
\text { del problema. }\end{array}$ & 7.51 & 5.93 & 7.69 \\
$\begin{array}{l}\text { Planificar la resolución identificando momento y tiempos } \\
\text { requeridos. }\end{array}$ & 7.80 & 7.40 & 7.35 \\
$\begin{array}{l}\text { Optimizar la selección y uso de materiales y dispositivos } \\
\text { tecnológicos disponibles para la implementación. }\end{array}$ & 8.44 & 7.96 \\
$\begin{array}{l}\text { Elaborar informes, planos recomendaciones y comunicarlos. } \\
\text { Controlar el proceso de ejecución. }\end{array}$ & 8.45 & 6.53 \\
\hline
\end{tabular}

Fuente: Elaboración propia

Capacidad de controlar y evaluar enfoques y estrategias para abordar eficazmente la resolución de los problemas

$\mathrm{Al}$ analizar los resultados correspondientes esta capacidad, en la tabla 6 , se observa que el componente con mayor nivel de requerimiento fue "Establecer supuestos, usar técnicas eficaces y estimar errores" (8.49). Y si bien el valor observado resultó ser el más alto (8.27) de todos los componentes, puede apreciarse que existe una brecha que marcaría la insatisfacción del sector empleador respecto de la formación de la competencia vinculada, ya que el valor del requerimiento se ubicó en (8.49).

Los componentes "Identificar lo que es relevante conocer y disponer de estrategias para adquirir conocimientos necesarios" y "Monitorear, evaluar y ajustar procesos de resolución de problemas" resultaron ser los que verificaron el menor nivel de adquisición por parte de los encuestados (7.78 y 7.80 respectivamente). 
Tabla 6. VO, VE y VR para los componentes de la capacidad "Controlar y Evaluar enfoques y estrategias para abordar eficazmente la resolución de problemas"

\begin{tabular}{lccc}
\hline Componentes de la capacidad & VO & VE & VR \\
\hline $\begin{array}{l}\text { Controlar el desempeño y saber cómo encontrar los recursos } \\
\text { necesarios para superar dificultades. }\end{array}$ & 8.04 & 7.18 & 8.16 \\
$\begin{array}{l}\text { Establecer supuestos, usar técnicas eficaces y estimar errores. } \\
\text { Monitorear, evaluar y ajustar procesos de resolución de }\end{array}$ & 8.27 & 7.95 & 8.49 \\
$\begin{array}{l}\text { problemas. } \\
\text { Identificar lo que es relevante conocer y disponer de } \\
\text { estrategias para adquirir conocimientos necesarios. }\end{array}$ & 7.78 & 8.11 & 8.24 \\
\hline
\end{tabular}

Fuente: Elaboración propia

\section{Índice IAC e IDC}

Como se señaló en los apartados precedentes, a partir de las medias se construyeron dos índices:

a) IAC: Se trata de un indicador de la calidad percibida que mide para cada componente la distancia entre las medias estadísticas del VO y el VE. De esta manera, se evalúa la satisfacción del graduado durante su proceso de formación, es decir, mide en qué medida las expectativas fueron satisfechas a través de las actividades formativas desarrolladas en los distintos espacios curriculares.

b) IDC: Este indicador mide de forma indirecta la satisfacción del sector empleador. El mismo tiene en cuenta la diferencia entre las medias estadísticas obtenidas entre el VO y el VR. Por lo tanto, mide a través de una variable del contexto (opinión del sector empleador) la necesidad de profundizar los resultados de los aprendizajes.

\section{Capacidad para identificar y formular problemas}

Los resultados que se presentan en la tabla 7 muestran que el IAC resultó satisfactorio para todos los componentes, con excepción de "Evaluar el contexto particular del problema e incluirlo en el análisis", ya que se verifica una leve insatisfacción (-0.05) en función de que la expectativa fue más alta que la percepción de los resultados de los aprendizajes. La mayor adecuación se observa para el componente "Identificar una situación problemática" (1.12). 
El IDC, por su parte, presenta resultados negativos, ya que con excepción del componente "Identificar una situación problemática", que muestra una superioridad de 0.41 , en el resto de los casos la relación se invierte. Se observa que los empleadores demandaron niveles más altos de desarrollo para los componentes "Identificación y organización de datos pertinentes a un problema", "Evaluación del contexto particular del problema para su inclusión en el análisis" y "Delimitación y formulación del problema de manera clara y precisa", cuyos índices fueron 0.58, 0.52 y 0.53 respectivamente.

Tabla 7. IAC e IDC para la capacidad "Identificar y formular problemas"

\begin{tabular}{llcc}
\hline \multicolumn{1}{c}{ Capacidad } & \multicolumn{1}{c}{ Componentes de la capacidad } & IAC & IDC \\
\hline $\begin{array}{l}\text { Identificar y } \\
\text { formular problemas }\end{array}$ & $\begin{array}{l}\text { Identificar situación problemática. } \\
\text { Identificar y organizar los datos pertinentes a } \\
\end{array}$ & 0.28 & -0.58 \\
& $\begin{array}{l}\text { unproblema. } \\
\text { Evaluar el contexto particular del problema e } \\
\text { incluirlo en el análisis. } \\
\end{array}$ & -0.05 & -0.52 \\
& $\begin{array}{l}\text { Delimitar el problema y formularlo de manera } \\
\text { clara y precisa. }\end{array}$ & 0.42 & -0.53 \\
\hline
\end{tabular}

Fuente: Elaboración propia

A partir de los índices obtenidos para la capacidad "Identificar y formular problemas", se observa que, en líneas generales, existe adecuación con las expectativas de formación que tienen los alumnos, en tanto que, en relación con el mercado de trabajo, los resultados de los aprendizajes se ubican en $75 \%$ de los casos, levemente por debajo de los requerimientos, y no satisfacen adecuadamente las expectativas del aquel (ver figura1). 


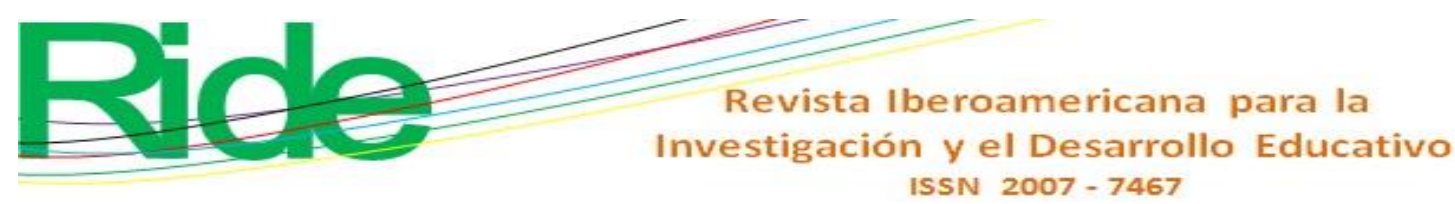

Figura 1. Brechas por cubrir para la capacidad "Identificar y formular problemas" en función de los índices IAC e IDC

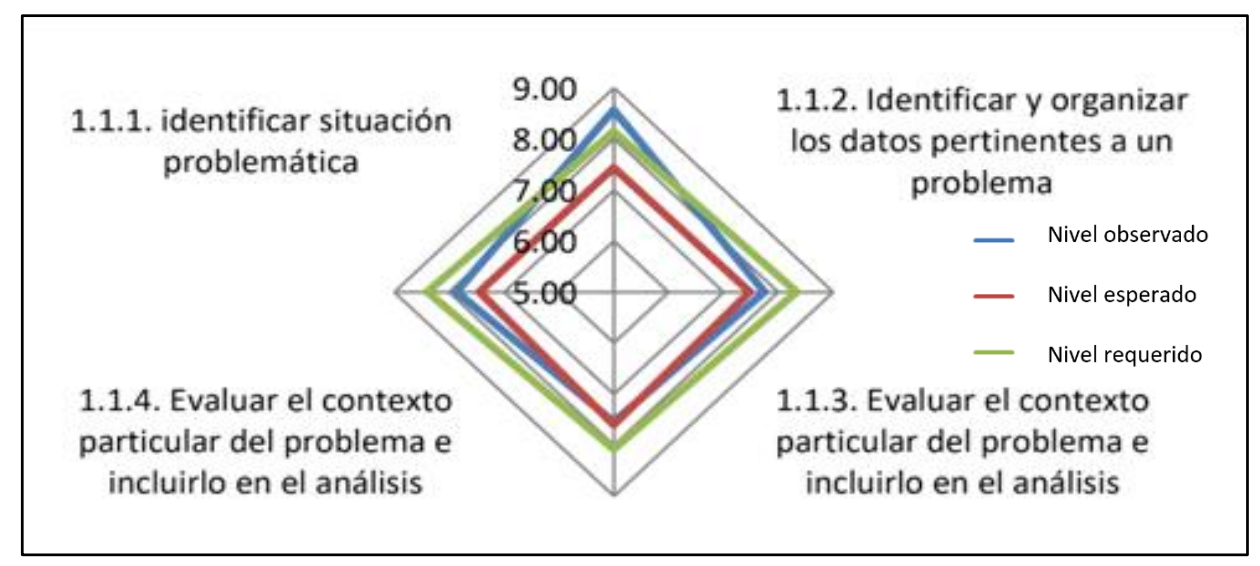

Fuente: Elaboración propia

\section{Capacidad para realizar búsqueda creativa de soluciones y seleccionar la alternativa más adecuada}

Al analizar el IAC (tabla 8) se advierte que el componente "Valorar el impacto sobre el medio ambiente y la sociedad, de las diversas alternativas de solución" no resultó satisfactorio, debido a que el valor obtenido fue de -0.37 ; resultado que estaría indicando que los graduados percibieron que los aprendizajes obtenidos fueron menores a sus expectativas. Este caso resulta significativo pues el IDC para el mismo componente se ubicó en 0.09, es decir, que el sector empleador no demandó un mayor nivel, sino que el requerimiento fue menor. Por el contrario, se observa un IDC negativo (-0.16) para los componentes "Generar diversas alternativas de solución a un problema ya formulado" y "Desarrollar criterios profesionales para la evaluación de las alternativas y selección la más adecuada a un contexto particular”, resultado que indicaría que las demandas laborales fueron mayores que los resultados de los aprendizajes adquiridos. 


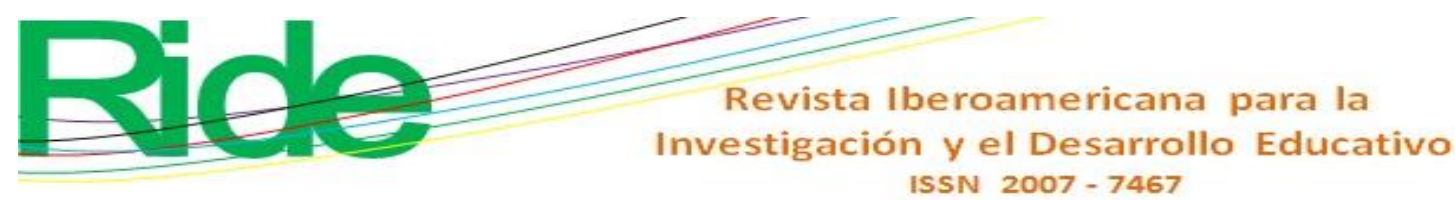

Tabla 8. IAC e IDC para la capacidad "Realizabúsqueda creativa de soluciones yseleccionar la alternativa más adecuada"

\begin{tabular}{llll}
\hline Capacidad & Componentes de la capacidad & IAC & IDC \\
\hline $\begin{array}{l}\text { Realizar búsqueda } \\
\text { creativa de }\end{array}$ & $\begin{array}{l}\text { Generar diversas alternativas de solución a un } \\
\text { poluciones y }\end{array}$ & 0.78 & -0.16 \\
$\begin{array}{l}\text { seleccionar la } \\
\text { alternativa más } \\
\text { adecuada }\end{array}$ & $\begin{array}{l}\text { Desarrollar criterios profesionales para la } \\
\text { evaluación de las alternativas y seleccionar la } \\
\text { más adecuada en un contexto particular. }\end{array}$ & 0.96 & -0.16 \\
& $\begin{array}{l} \\
\text { Valorar el impacto sobre el medio ambiente y } \\
\text { la sociedad, de las diversas alternativas de } \\
\text { solución. }\end{array}$ & -0.37 & \multirow{2}{*}{0.09} \\
& & & \\
\hline
\end{tabular}

Fuente: Elaboración propia

En la figura 2 se representan las brechas porcubrir para adecuar tanto la satisfacción de los estudiantes como la del sector empleador. También, como en el caso anterior, el IAC presenta un buen nivel de adecuación, mientras que el IDC estaría indicando la necesidad de plantear un trabajo de mejora del nivel desarrollo de las capacidades a fin de contribuir a la adquisición de las competencias según los requerimientos del sector empleador. 


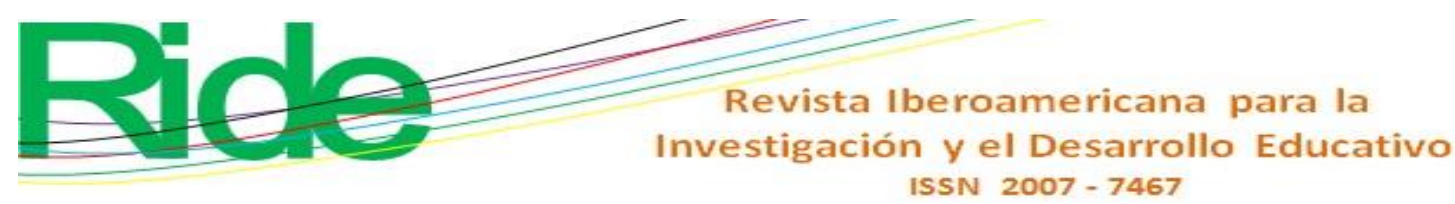

Figura 2. Brechas por cubrir para la capacidad "Realizar búsqueda creativa de soluciones y seleccionar la alternativa más adecuada" en función de los IAC e IDC

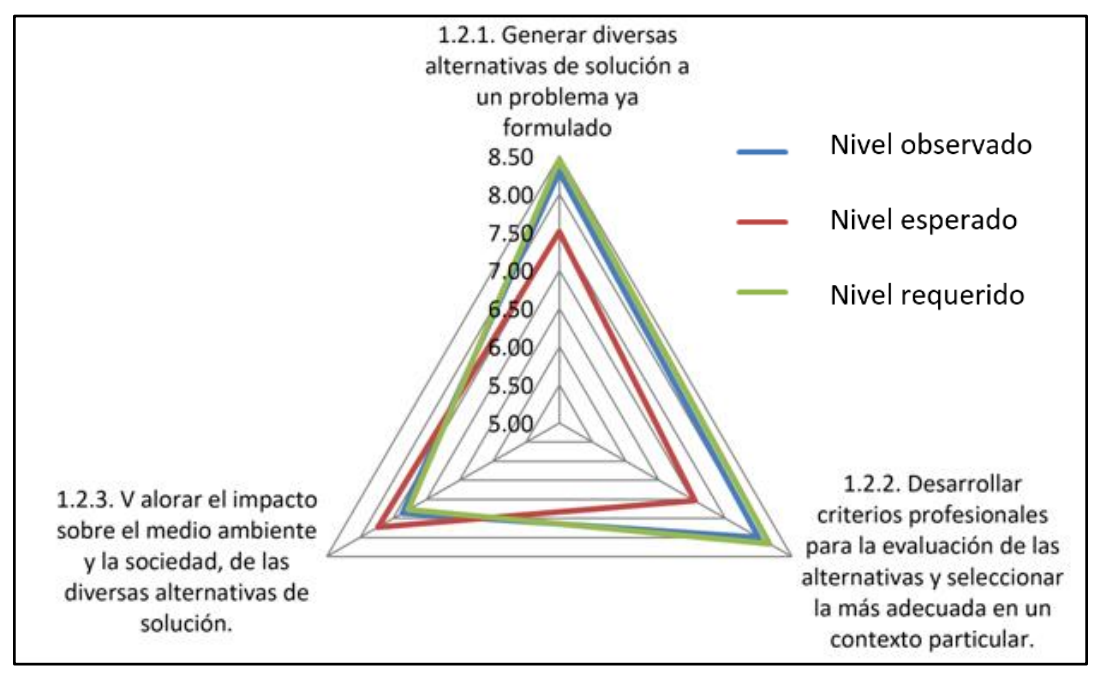

Fuente: Elaboración propia

\section{Capacidad para implementar tecnológicamente una alternativa de solución}

Respecto a esta capacidad, el IAC resultó ser satisfactorio en todos los casos (ver tabla 9), con niveles de adecuación muy significativos, como en el caso de "Realizar el diseño y modelado de una solución tecnológica", "Elaborar informes, planos, recomendaciones y comunicarlos" y "Controlar el proceso de ejecución", cuyos índices resultaron ser de 1.58 y 1.65 y 1.42, respectivamente. Sin embargo, al analizar los resultados del IDC, se advierte que solo existe una equiparación o superioridad para los componentes "Incorporar a un diseño las dimensiones relevantes al contexto del problema" (0.07) y "Controlar el proceso de ejecución" (0). 


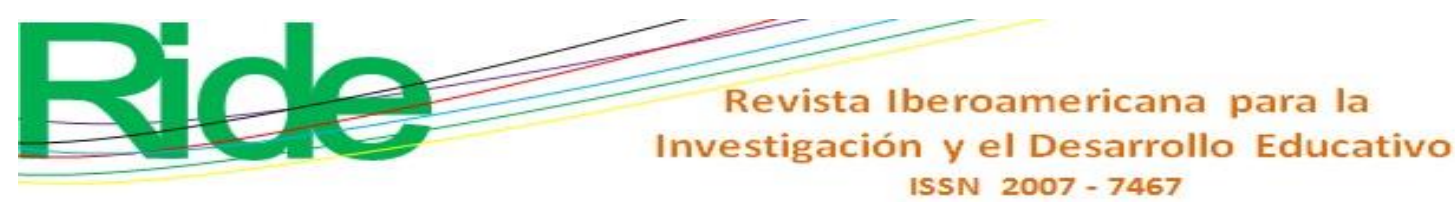

Tabla 9. IAC e IDC para la capacidad "Implementar tecnológicamente una alternativa de solución"

\begin{tabular}{llcc}
\hline Capacidad & Componentes de la capacidad & IAC & IDC \\
\hline $\begin{array}{l}\text { Implementar } \\
\text { tecnológicamente } \\
\text { una alternativa de } \\
\text { solución }\end{array}$ & $\begin{array}{l}\text { Realizar el diseño y modelado de una solución } \\
\text { tecnológica. }\end{array}$ & 1.58 & -0.18 \\
& $\begin{array}{l}\text { Incorporar a un diseño las dimensiones } \\
\text { relevantes al contexto del problema. } \\
\text { Planificar la resolución identificando momento } \\
\text { y tiempos requeridos. }\end{array}$ & 0.64 & -0.16 \\
& $\begin{array}{l}\text { Optimizar la selección y uso de materiales y } \\
\text { dispositivos tecnológicos disponibles para la }\end{array}$ & 0,07 & $-0,09$ \\
& $\begin{array}{l}\text { implementación. } \\
\text { Elaborar informes, planos recomendaciones y } \\
\text { comunicarlos. }\end{array}$ & 1,65 & $-0,26$ \\
& Controlar el proceso de ejecución. & 1,42 & 0 \\
\hline
\end{tabular}

Fuente: Elaboración propia

Por los resultados obtenidos en el IAC, se puede afirmar que existe un buen nivel de adecuación entre las expectativas y los niveles de formación alcanzados. Sin embargo, el IDC presenta $66 \%$ de déficits para los componentes, es decir, existen brechas, aunque no muy significativas, entre lo observado por los graduados y el nivel de requerimiento del mercado laboral, tal y como puede observarse en la figura 3. 


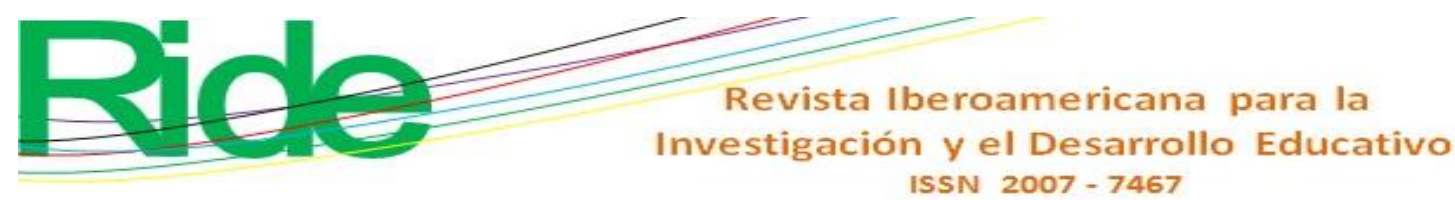

Figura 3. Brechas por cubrir para la capacidad "Implementar tecnológicamente una alternativa de solución" en función de los IAC e IDC

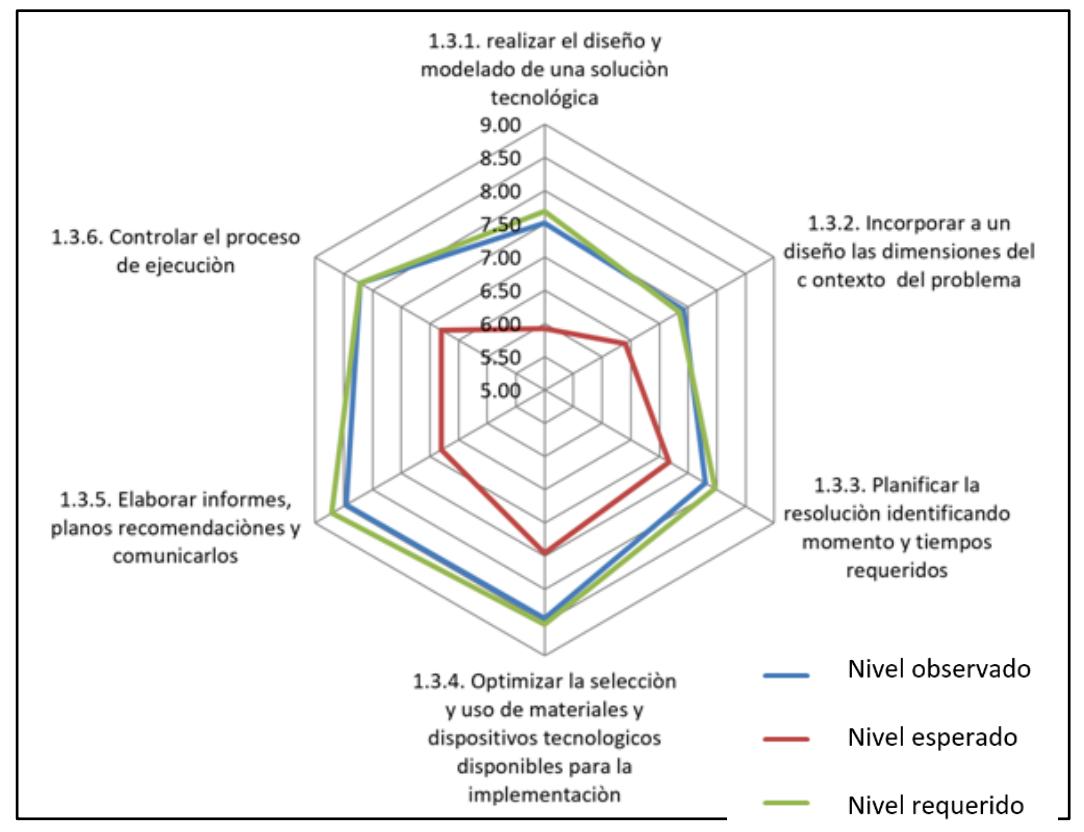

Fuente: Elaboración propia

\section{Capacidad para controlar y evaluar enfoques y estrategias para abordar eficazmente la resolución de problemas}

El IAC presenta para esta capacidad falta de adecuación entre el valor esperado y el observado solo para el componente "Identificar lo que es relevante conocer y disponer de estrategias para adquirir conocimientos necesarios"; en el IDC, mientras tanto, existen disconformidades en todos los componentes (véase tabla 10). 
Tabla 1. Índices IAC e IDC para la capacidad "Controlar y evaluar enfoques y estrategias para abordar eficazmente la resolución de los problemas"

\begin{tabular}{|c|c|c|c|}
\hline Capacidad & Componente de la capacidad & IAC & IDC \\
\hline $\begin{array}{l}\text { Controlar y evaluar } \\
\text { enfoques y estrategias } \\
\text { para abordar }\end{array}$ & $\begin{array}{l}\text { Controlar el desempeño y saber cómo } \\
\text { encontrar los recursos necesarios para superar } \\
\text { dificultades. }\end{array}$ & 0.86 & -0.12 \\
\hline $\begin{array}{l}\text { eficazmente la } \\
\text { resolución de los }\end{array}$ & $\begin{array}{l}\text { Establecer supuestos, usar técnicas eficaces y } \\
\text { estimar errores. }\end{array}$ & 0.32 & -0.22 \\
\hline \multirow[t]{2}{*}{ problemas } & $\begin{array}{l}\text { Monitorear evaluar y ajustar procesos de } \\
\text { resolución de problemas. }\end{array}$ & 0.16 & -0.40 \\
\hline & $\begin{array}{l}\text { Identificar lo que es relevante conocer y } \\
\text { disponer de estrategias para adquirir } \\
\text { conocimientos necesarios. }\end{array}$ & -0.33 & -0.46 \\
\hline
\end{tabular}

Fuente: Elaboración propia

En la figura 4 se visualizan las brechas por cubrir a partir de ambos índices; y se advierte cómo el IDC muestra que los aprendizajes alcanzados durante la carrera para todos los componentes no fueron suficientes para satisfacer el requerimiento del mercado de trabajo.

Figura 4. Brechas por cubrir para lacapacidad "Controlar y evaluar enfoques y estrategias para abordar eficazmente la resolución de los problemas"

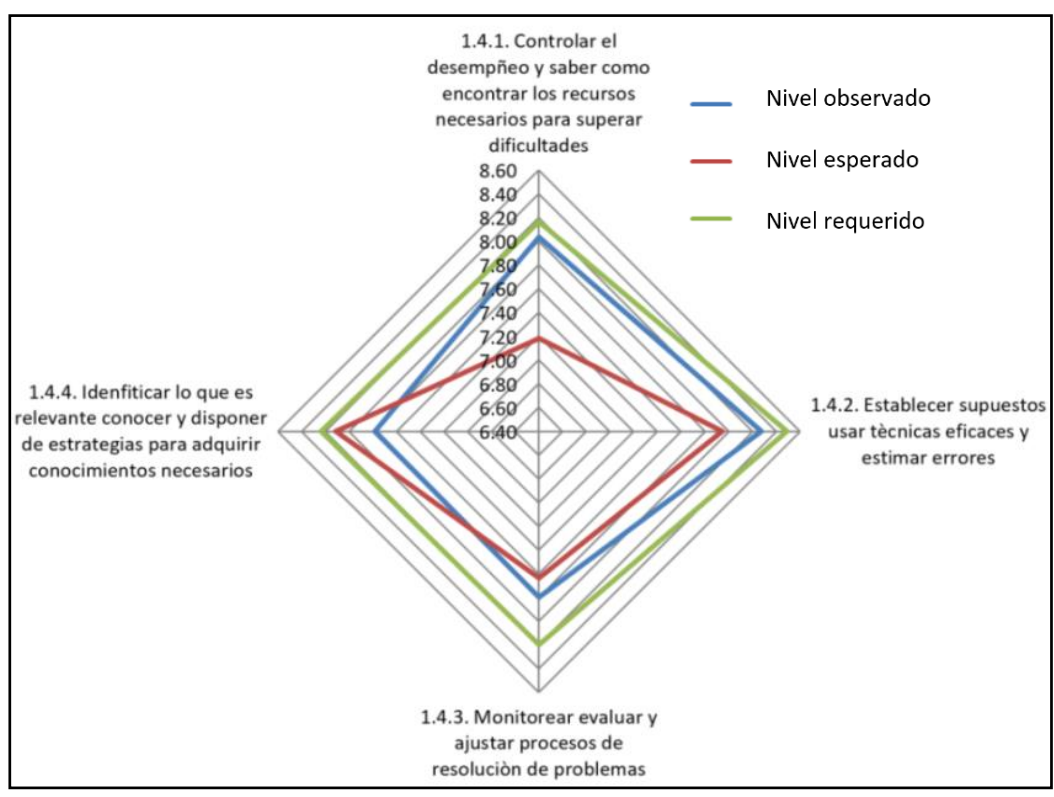

Fuente: Elaboración propia 


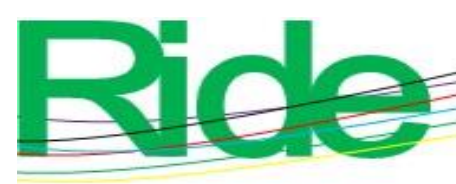

Revista Iberoamericana para la

Investigación y el Desarrollo Educativo

ISSN 2007 - 7467

\section{Discusión}

En la tabla 11 se presentan los resultados correspondientes a la adecuación entre las expectativas y la percepción del servicio educativo (IAC). Los datos fueron ordenados para cada capacidad con criterio decreciente y se advierte que existe satisfacción en $83 \%$ de los 17 componentes evaluados. Solo los aprendizajes inherentes, a saber: a) Evaluar el contexto particular del problema e incluirlo en el análisis", b) "Valorar el impacto sobre el medio ambiente y la sociedad, de las diversas alternativas de solución” y c) Identificar lo que es relevante conocer y disponer de estrategias para adquirir conocimientos necesarios, no alcanzaron a satisfacer las expectativas de los encuestados. En función de los valores obtenidos la brecha no resulta significativa; y si bien el diseño curricular vigente se enmarca en el enfoque de contenidos, se puede inferir que las estrategias desarrolladas durante la implementación de la actual propuesta curricular estarían garantizando un adecuado nivel de adquisición de las capacidades desde las expectativas de los graduados.

Merece un especial señalamiento la capacidad para implementar tecnológicamente una alternativa de solución tecnológica, ya que sus componentes ("Incorporar a un diseño las dimensiones relevantes al contexto del problema, planificar la resolución identificando momento y tiempos requeridos", "Optimizar la selección y uso de materiales y dispositivos tecnológicos",

"Elaborar informes, planos, recomendaciones y comunicarlos" y "Controlar el proceso de ejecución”) obtuvieron un ponderación positiva para el IAC en todos los casos.

Es importante, asimismo, destacar el interés de los graduados en contar con estrategias para adquirir conocimientos, ya que la expectativa superó la percepción de su adquisición. Se trata de una capacidad directamente asociada a las competencias de aprendizaje continuo y a la autonomía en los estudios que resultan significativas para un adecuado desarrollo profesional. Las mismas son consideradas transversales, ya que por sus características no se adquieren en un espacio curricular determinado, sino que se desarrollan gradualmente a lo largo de toda la carrera, aspecto para considerar particularmente al momento de definir el nuevo diseño curricular. 
Tabla 11. Nivel de adecuación y disconformidad en función del IAC

\begin{tabular}{|c|c|c|c|}
\hline \multirow[t]{2}{*}{ Capacidad } & \multirow[t]{2}{*}{ Componente de la capacidad } & \multicolumn{2}{|c|}{ IAC } \\
\hline & & Adecuación & Disconformidad \\
\hline \multirow[t]{4}{*}{$\begin{array}{l}\text { Identificar y } \\
\text { Formular Problemas }\end{array}$} & $\begin{array}{l}\text { Identificar una situación } \\
\text { problemática }\end{array}$ & 1,12 & \\
\hline & $\begin{array}{l}\text { Delimitar el problema y } \\
\text { formularlo de manera clara y pre }\end{array}$ & 0,42 & \\
\hline & $\begin{array}{l}\text { Identificar y organizar los datos } \\
\text { pertinentes a un problema }\end{array}$ & 0,28 & \\
\hline & $\begin{array}{l}\text { Evaluar el contexto particular del } \\
\text { problema e incluirlo en el análisis }\end{array}$ & & $-0,05$ \\
\hline \multirow[t]{3}{*}{$\begin{array}{l}\text { Realizar búsqueda } \\
\text { creativa de } \\
\text { soluciones y } \\
\text { seleccionar la } \\
\text { alternativa más } \\
\text { adecuada }\end{array}$} & $\begin{array}{l}\text { Desarrollar criterios profesionales } \\
\text { para la evaluación de las } \\
\text { alternativas y seleccionar la más } \\
\text { adecuada en un contexto } \\
\text { particular }\end{array}$ & 0,96 & \\
\hline & $\begin{array}{l}\text { Generar diversas alternativas de } \\
\text { solución a un problema ya } \\
\text { formulado }\end{array}$ & 0,78 & \\
\hline & $\begin{array}{l}\text { Valorar el impacto sobre el medio } \\
\text { ambiente y la sociedad, de las } \\
\text { diversas alternativas de solución }\end{array}$ & & $-0,37$ \\
\hline \multirow{6}{*}{$\begin{array}{l}\text { Implementar } \\
\text { tecnológicamente } \\
\text { una alternativa de } \\
\text { solución }\end{array}$} & $\begin{array}{l}\text { Elaborar informes, planos } \\
\text { recomendaciones y comunicación }\end{array}$ & 1,65 & \\
\hline & $\begin{array}{l}\text { Realizar el diseño y modelado de } \\
\text { una solución tecnológica }\end{array}$ & 1,58 & \\
\hline & Controlar el proceso de ejecución & 1,42 & \\
\hline & $\begin{array}{l}\text { Incorporar a un diseño las } \\
\text { dimensiones relevantes al } \\
\text { contexto del problema }\end{array}$ & 1,02 & \\
\hline & $\begin{array}{l}\text { Optimizar la selección y uso de } \\
\text { materiales y dispositivos } \\
\text { tecnológicos disponibles para la } \\
\text { implementación }\end{array}$ & 0,99 & \\
\hline & $\begin{array}{l}\text { Planificar la resolución } \\
\text { identificando momento y tiempos } \\
\text { requeridos }\end{array}$ & 0,64 & \\
\hline Controlar y evaluar & Controlar el desempeño y saber & 0,86 & \\
\hline
\end{tabular}




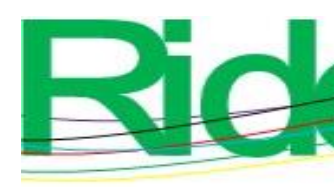

Revista Iberoamericana para la

Investigación y el Desarrollo Educativo

ISSN 2007 - 7467

\begin{tabular}{|c|c|c|c|}
\hline \multirow{4}{*}{$\begin{array}{l}\text { enfoques y } \\
\text { estrategias para } \\
\text { abordar eficazmente } \\
\text { la resolución de los } \\
\text { problemas }\end{array}$} & $\begin{array}{l}\text { cómo encontrar los recursos } \\
\text { necesarios para superar } \\
\text { dificultades }\end{array}$ & & \\
\hline & $\begin{array}{l}\text { Establecer supuestos, usar } \\
\text { técnicas eficaces y estimar errores }\end{array}$ & 0,32 & \\
\hline & $\begin{array}{l}\text { Monitorear evaluar y ajustar } \\
\text { procesos de resolución de } \\
\text { problemas }\end{array}$ & 0,16 & \\
\hline & $\begin{array}{l}\text { Identificar lo que es relevante } \\
\text { conocer y disponer de estrategias } \\
\text { para adquirir conocimientos } \\
\text { necesarios }\end{array}$ & & $-0,33$ \\
\hline
\end{tabular}

Fuente: Elaboración propia

El IDC, que en la tabla 12, también ha sido ordenado para cada capacidad con criterio decreciente, muestra un desempeño diferente respecto del IAC, ya que solo $23.5 \%$ de los componentes presentaron un índice de superioridad muy baja o neutra, como en el caso del componente "Controlar el proceso de ejecución"(0).

Se observa que el sector empleador advirtió déficits para $76.5 \%$ de los componentes; por su número y magnitud, el más significativo fue "Identificar y formular problemas", lo cual resulta ser una cuestión liminar de la competencia que se analiza, ya que si el profesional no cuenta con las herramientas para identificar y formular técnicamente una situación problemática, deviene en una dificultad ulterior tanto para la búsqueda de soluciones como para su posterior implementación, control y evaluación. No obstante, resulta interesante que, en relación con esta capacidad, el componente de "Identificar una situación problemática" en el IAC obtuvo el valor de adecuación más alto (1.12) y en el IDC el mayor índice de superioridad (0.41).

Los déficits identificados a través del IDC advierten sobre la necesidad de realizar un exhaustivo trabajo de reflexión y ajuste al momento de pensar en la innovación curricular hacia un diseño por competencias, con el objeto de asegurar que se garantice un trabajo coordinado y orientado al desarrollo de las capacidades entre los distintos espacios curriculares que se definan. 
Tabla 12. Niveles de superioridad y déficits en función del IDC

\begin{tabular}{|c|c|c|c|}
\hline \multirow[t]{2}{*}{ Capacidad } & \multirow[t]{2}{*}{$\begin{array}{c}\text { Componentes de la } \\
\text { capacidad }\end{array}$} & \multicolumn{2}{|c|}{ IDC } \\
\hline & & Superioridad & $\begin{array}{l}\text { Déficit para } \\
\text { sector } \\
\text { empleador }\end{array}$ \\
\hline \multirow[t]{4}{*}{$\begin{array}{l}\text { Identificar y formular } \\
\text { problemas }\end{array}$} & $\begin{array}{l}\text { Identificar una situación } \\
\text { problemática. }\end{array}$ & 0.41 & \\
\hline & $\begin{array}{l}\text { Identificar y organizar } \\
\text { los datos pertinentes a } \\
\text { un problema. }\end{array}$ & & -0.58 \\
\hline & $\begin{array}{l}\text { Delimitar el problema y } \\
\text { formularlo de manera } \\
\text { clara y precisa. }\end{array}$ & & -0.53 \\
\hline & $\begin{array}{l}\text { Evaluar el contexto } \\
\text { particular del problema } \\
\text { e incluirlo en el análisis. }\end{array}$ & & -0.52 \\
\hline \multirow{3}{*}{$\begin{array}{l}\text { Realizar búsqueda } \\
\text { creativa de soluciones y } \\
\text { seleccionar la } \\
\text { alternativa más } \\
\text { adecuada }\end{array}$} & $\begin{array}{l}\text { Valorar el impacto sobre } \\
\text { el medio ambiente y la } \\
\text { sociedad, de las diversas } \\
\text { alternativas de solución. }\end{array}$ & 0.09 & \\
\hline & $\begin{array}{l}\text { Generar diversas } \\
\text { alternativas de solución } \\
\text { a un problema ya } \\
\text { formulado. }\end{array}$ & & -0.16 \\
\hline & $\begin{array}{l}\text { Desarrollar criterios } \\
\text { profesionales para la } \\
\text { evaluación de las } \\
\text { alternativas y } \\
\text { seleccionar la más } \\
\text { adecuada en un contexto } \\
\text { particular. }\end{array}$ & & -0.16 \\
\hline \multirow[t]{2}{*}{$\begin{array}{l}\text { Implementar } \\
\text { tecnológicamente una } \\
\text { alternativa de solución }\end{array}$} & $\begin{array}{l}\text { Incorporar a un diseño } \\
\text { las dimensiones } \\
\text { relevantes al contexto } \\
\text { del problema. }\end{array}$ & 0.07 & \\
\hline & Controlar el proceso de & 0 & \\
\hline
\end{tabular}




\begin{tabular}{|c|c|c|}
\hline & ejecución. & \\
\hline & $\begin{array}{l}\text { Elaborar informes, } \\
\text { planos recomendaciones } \\
\text { y comunicarlos. }\end{array}$ & -0.26 \\
\hline & $\begin{array}{l}\text { Realizar el diseño y } \\
\text { modelado de una } \\
\text { solución tecnológica. }\end{array}$ & -0.18 \\
\hline & $\begin{array}{l}\text { Planificar la resolución } \\
\text { identificando momento } \\
\text { y tiempos requeridos. }\end{array}$ & -0.16 \\
\hline & $\begin{array}{l}\text { Optimizar la selección y } \\
\text { uso de materiales y } \\
\text { dispositivos } \\
\text { tecnológicos disponibles } \\
\text { para la implementación. }\end{array}$ & -0.09 \\
\hline \multirow[t]{4}{*}{$\begin{array}{l}\text { Controlar y evaluar } \\
\text { enfoques y estrategias } \\
\text { para abordar } \\
\text { eficazmente la } \\
\text { resolución de los } \\
\text { problemas }\end{array}$} & $\begin{array}{l}\text { Identificar lo que es } \\
\text { relevante conocer y } \\
\text { disponer de estrategias } \\
\text { para adquirir } \\
\text { conocimientos } \\
\text { necesarios. }\end{array}$ & -0.46 \\
\hline & $\begin{array}{l}\text { Monitorear evaluar y } \\
\text { ajustar procesos de } \\
\text { resolución de problema. }\end{array}$ & -0.40 \\
\hline & $\begin{array}{l}\text { Establecer supuestos, } \\
\text { usar técnicas eficaces y } \\
\text { estimar errores. }\end{array}$ & -0.22 \\
\hline & $\begin{array}{l}\text { Controlar el desempeño } \\
\text { y saber cómo encontrar } \\
\text { los recursos necesarios } \\
\text { para superar } \\
\text { dificultades. }\end{array}$ & -0.12 \\
\hline
\end{tabular}

Fuente: Elaboración propia 


\section{Conclusiones}

A partir del mapa funcional de competencias desarrollado por el Confedi para la formación de ingenieros en Argentina, desde el IIT\&E se ha estructurado un modelo que mide su nivel de adquisición desde la perspectiva de la evaluación de la calidad de los servicios educativos.

El modelo permitió construir dos índices estadísticos a partir de juicios de graduados y obtener un criterio ponderado de su satisfacción con los aprendizajes alcanzados, así como un ordenamiento de los déficits en función de las experiencias laborales por las que transitaron.

El IAC y el IDC aportan información sobre la adecuación de las expectativas de los graduados y la superioridad o déficits en relación con los requerimientos del mercado de trabajo que resulta estratégica para la toma de decisiones durante procesos de innovación que requieran, desde la perspectiva de la calidad educativa, la satisfacción de los usuarios.

Se observa que los juicios de valor respecto a la cobertura de las expectativas personales no siempre resultaron consistentes con las experiencias concretas en materia de inserción laboral, ya que se dieron casos en los que el IAC resultó de adecuación y el IDC indicó déficit. En este sentido, el IAC obtuvo $83 \%$ de adecuación para los 17 componentes, en tanto que el IDC solo resultó con un indicador de superioridad en $23.5 \%$ de los casos; es decir, que para el sector empleador $76.5 \%$ de los componentes presentó déficits. Esta cuestión advierte acerca de la necesidad de trabajar con métodos que triangulen datos para dotar de validez y confiabilidad a los constructos metodológicos que los sustenten. El desarrollo de un segundo instrumento que mida exclusivamente los requerimientos del sector empleador y cuyos resultados se comparen con el IDC puede ser una alternativa válida.

Si bien se trata de resultados parciales, se puede adelantar que las carreras de la Facultad de Ingeniería de la UNLZ, en general, cubren desde la perspectiva de los graduados las expectativas respecto a la adquisición de las capacidades asociadas a la competencia "Identificar, formular y resolver problemas de ingeniería, como así mismo concebir, diseñar y desarrollar proyectos de ingeniería", a pesar de que sus experiencias laborales han puesto en evidencia ciertos déficits. 

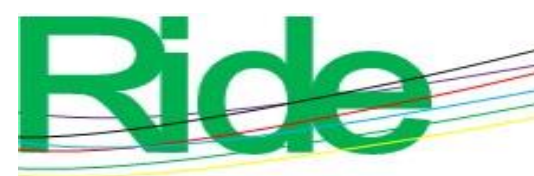

\section{Revista Iberoamericana para la Investigación y el Desarrollo Educativo ISSN 2007 - 7467}

Por último, el análisis se ha circunscripto solo a una de las cinco competencias tecnológicas definidas para la formación de los ingenieros, por lo tanto, queda pendiente la elaboración de índices para las restantes cuatro competencias de naturaleza tecnológica, al igual que para las cinco de orden social, político y actitudinal. Si bien al momento se cuentan con datos que permiten inferir un diagnóstico respecto de la competencia analizada, es necesario contar con el análisis completo del actual perfil formativo para confeccionar el mapa funcional, que incluya a la totalidad de competencias del ingeniero y se las vincule con los índices obtenidos. Esta matriz permitirá a la Facultad de Ingeniería de la UNLZ inferir las brechas por cubrir en el nuevo diseño curricular de sus carreras, evaluar la magnitud del cambio y coordinar con los responsables de su implementación la articulación de las actividades que aseguren la calidad de los resultados del nuevo diseño y garantice tanto la satisfacción de los alumnos por la enseñanza que reciben como la cobertura de las expectativas y necesidades del sector productivo.

Se puede concluir que el modelo desarrollado ha puesto a disposición de la institución una herramienta que, además de medir los resultados de los aprendizajes desde la perspectiva de sus graduados y valorar su nivel de satisfacción con las competencias adquiridas, contribuye a ponderar las brechas formativas en función de los requerimientos del mercado de trabajo. 


\section{Referencias}

Comisión Nacional de Evaluación y Acreditación Universitaria [Coneau]. (2015). Ingeniería. Impacto en la calidad educativa. Buenos Aires, Argentina: Comisión Nacional de Evaluación y Acreditación Universitaria.

Consejo Federal de Decanos de Ingeniería de la República Argentina [Confedi]. (2006). Primer Acuerdo sobre Competencias Genéricas. Villa Carlos Paz, Argentina: Consejo Federal de Decanos de Ingeniería de la República Argentina.

Consejo Federal de Decanos de Ingeniería de la República Argentina [Confedi]. (2017). Documento Final de Propuesta de Nuevos Estándares de Carreras de Ingeniería. Paraná, Argentina: Consejo Federal de Decanos de Ingeniería de la República Argentina.

De la Cruz Flores, G. y Abreu Hernández, L. (2014). Rúbricas y autorregulación: pautas para promover una cultura de la autonomía de la formación profesional terciaria. Revista de Docencia Universitaria, 12(1), 31-48.

Ministros Europeos. (1999). Declaración de Bolonia. Declaración conjunta de los ministros europeos de educación. Bolonia, Italia: Ministros Europeos.

Duque Oliva, E., \& Chaparro Pinzón, C. (2017). Medición de la percepción de la calidad del servicio de educación por parte de los estudiantes de la UPTC Duitama. Criterio Libre, 10(16), 159-192.

Duque Oliva, E. y Diosa Gómez, Y. (2014). Evolución conceptual de los modelos de medición de la percepción de la calidad del servicio: una mirada desde la educación superior. Suma de negocios, $5(12)$ 180-191.Recuperado de https://www.sciencedirect.com/science/article/pii/S2215910X14700400.

Herrero, R., González, I. y Marín, V. (2015). Formación centrada en competencias estudiantiles en Educación Superior.Revista de Ciencias Sociales XXI,(4), 461-478. Recuperado de https://www.researchgate.net/publication/292616948_Formacion_centrada_en_competenc $\underline{\text { ias_estudiantiles_en_educacion_superior. }}$

Huerta, M. (2014). Formación por competencias a través del aprendizaje estratégico. Lima, Perú: Editorial San Marcos. 


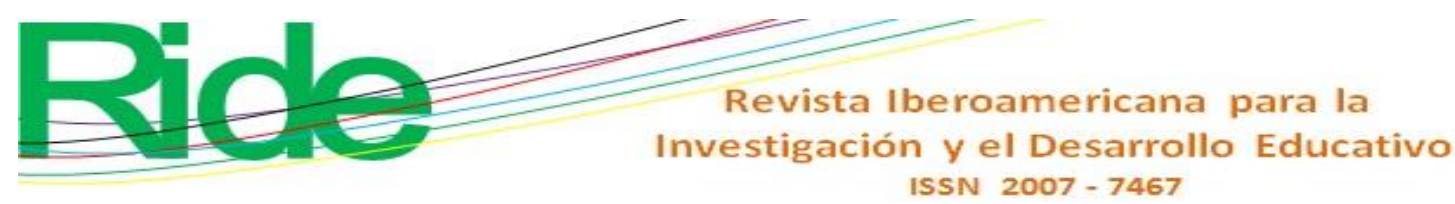

Martínez, M., Blanco, M. y Castán, J. (2013). Las dimensiones de la calidad del servicio percibida en entornos virtuales de formación superior. Revista de la Universidad y Sociedad del Conocimiento, 10, 89-106. Recuperado de doi.10.7238/rusc.v10i1.1411.

Pérez, L., Vilariño, C. y Ronda, G., (2017). El cambio organizacional como herramienta para coadyuvar con la implementación de la estrategia. Ingeniería Industrial,38(3), 223332.Recuperado de http://scielo.sld.cu/pdf/rii/v38n3/rii100317.pdf.

Perrenoud, P. (2013). Construir competencias desde la escuela. Madrid, España: Editorial JCSAEZ.

Tejada, J. (2012). La alternancia de contexto para la adquisición de competencias profesionales en escenarios complementarios de Educación Superior: Marco y Estrategia. EducaciónXXI, 15(2), 17-40. Recuperado de doi.org/10.5944/educxx1.15.2.125.

Tobón, S. (2013). Formación integral y competencias. Bogotá, Colombia: Eco Ediciones.

Tobón, S., Pimienta, J. y García, J. (2012). Secuencias didácticas: Aprendizaje y evaluación de competencias. México: Pearson Educación.

Ya-Hui,S., Li-Yia, F., Chao-Chin,Y. and Tzu-Ling, C. (2012). How teachers support university students' lifelong learning development for sustainable futures: The student's perspective. Futures, $\quad 44(2), \quad 158-165 . \quad$ Retrieved from https://www.sciencedirect.com/science/article/pii/S0016328711002473. 